\title{
Uncertainty Quantification for Modeling Pulsed Laser Ablation of Aluminum Considering Uncertainty in the Temperature-Dependent Absorption Coefficient
}

\author{
Yeqing Wang $^{1 *}$, Getachew K. Befekadu ${ }^{2}$, Hongtao Ding ${ }^{3}$, David W. Hahn ${ }^{1}$ \\ ${ }^{1}$ Department of Mechanical and Aerospace Engineering, University of Florida, Gainesville, FL \\ 32611, USA \\ ${ }^{2}$ NRC/Air Force Research Laboratory \& Department of Mechanical and Aerospace Engineering, \\ University of Florida - REEF, Shalimar, FL 32579, USA \\ ${ }^{3}$ Department of Mechanical and Industrial Engineering, University of Iowa, Iowa City, IA \\ 52246, USA
}

\begin{abstract}
In this paper, an extension of the result of Wang et al. ("Modeling pulsed laser ablation of aluminum with finite element analysis considering material moving front," Int. J. Heat \& Mass Transfer, 113, 1246-1253, 2017) concerning the problem of uncertainty quantification for pulsed laser ablation (PLA) of aluminum is considered, when the source of uncertainty is due to an inherent randomness of the temperature-dependent absorption coefficient. In particular, we use a generalized polynomial chaos (gPC) method to incorporate the parameter uncertainty for the temperature-dependent absorption coefficient within the representation of the laser heat conduction phenomena. Furthermore, numerical simulation studies for the PLA of aluminum, with nanosecond Nd:YAG $266 \mathrm{~nm}$ pulsed laser, that demonstrate the proposed $\mathrm{gPC}$ predictions

*Corresponding author, tel: +1-319-930-9956; e-mail address: yeqwang@reef.ufl.edu
\end{abstract}

(C) 2017. This manuscript version is made available under the Elsevier user license http://www.elsevier.com/open-access/userlicense/1.0/ 
are presented. Finally, a sensitivity study is performed to identify whether small changes in the lower and/or upper parameter values of the absorption coefficient provide the most variance in the thermal and ablation responses.

Keywords: Uncertainty quantification (UQ), Pulsed laser ablation (PLA), Absorption coefficient, Generalized polynomial chaos (gPC) method, Finite element analysis (FEA)

\section{Introduction}

In recent years, a number of research efforts have been devoted to understand the working mechanisms and develop accurate simulation models for laser ablation of solid materials [1-9]. Despite these efforts, the problem of uncertainty quantification (UQ) for laser ablation of solid materials, when the sources of uncertainty are due to (inherently stochastic) variability of material and optical properties of target materials at various elevated temperatures, is not sufficiently understood or addressed, while recognizing their critical impact on guiding experimental efforts and advanced manufacturing. This further necessitates the need for developing efficient UQ methods for laser ablation of solid materials that establish confidence intervals in the computed temperature predictions and/or ablation response, the assessment of the suitability of model formulations for laser ablation of solid materials and/or the support of decision-making analysis. In this paper, we extend our recent work [8] to address the problem of UQ for pulsed laser ablation (PLA) of aluminum, when the source of uncertainty is due to an inherent randomness of the temperature-dependent absorption coefficient. In particular, we use a generalized polynomial chaos ( $\mathrm{gPC}$ ) method to incorporate the parameter uncertainty of the 
temperature-dependent absorption coefficient within the representation of the laser heat conduction phenomena. Note that the fundamental concept, where the gPC expansions are used for representing random fields and/or stochastic variables, is to consider the uncertainty as generating a new stochastic dimension and observing the solutions as being dependent on this dimension [10-15]. A convergent expansion along the new stochastic dimension is then sought in terms of a set of orthogonal basis functions, whose coefficients can be used to characterize and quantify the uncertainty.

Here it is worth mentioning that the gPC based method has been extensively used for UQ in engineering problems of solid and fluid mechanics (e.g., see Refs. $[12,16]$ in the context of elastic structures; see Ref. [17] in the context of flow through porous media; and see Refs. [18, 19] in the context of thermal problems). The main motivation behind the PC expansions includes the suitability for models expressed in terms of a set of coupled partial differential equations, the ability to deal with situations exhibiting steep nonlinear dependence of the solution on random model data, and the promise of obtaining efficient and accurate estimates of uncertainty. Moreover, such an information provides a format that permits itself to be readily used to probe the dependence of specific observables on particular components of the input data and/or to design experiments in order to better calibrate and test the validity of postulated model representation. In this paper, numerical simulation studies for the PLA of aluminum, with nanosecond Nd:YAG $266 \mathrm{~nm}$ pulsed laser, that demonstrate the proposed gPC predictions are presented. Moreover, a sensitivity study is performed to identify whether small variations in the lower and/or upper parameter values of the absorption coefficient provide the most variance in the thermal and ablation response and also integrate the effect of uncertainty ranges of the parameters on the thermal and ablation response. 
The remainder of this paper is organized as follows. In Section 2, we outline the mathematical model of the laser heat conduction that accounts for the uncertainty in the absorption coefficient. The uncertainty is incorporated into the laser heat conduction through gPC expansions. In Section 3, the temperature-dependent material properties and the absorption coefficient uncertainty used for the UQ of the PLA model are presented. Section 4 briefly discusses the computational method used, where such a method captures the coupling between the material progressive surface recession and the laser heat conduction that also considers the material parameter uncertainties. Results and discussions demonstrating the applicability of the gPC based UQ for the PLA of aluminum are given in Section 5. Finally, our findings are summarized in Section 6.

\section{Mathematical Model for Laser Heat Conduction Considering Uncertainty in the Temperature-dependent Absorption Coefficient}

In the absence of convective and radiative heat exchanges, the thermal response of the solid material is governed by the following energy balance equation $[1,20,21]$

$$
\rho C_{p}\left(\frac{\partial T}{\partial t}-\dot{s} \frac{\partial T}{\partial z}\right)-\nabla \cdot(k \nabla T)=\dot{q},
$$

where $T$ is the temperature, the dependent variables are $t$ (time) and $z$, the spatial coordinate normal to the material surface (see also Fig. 1), and the material properties are $\rho$, the solid density, $C_{p}$, the specific heat. $s \square$ is the surface recession rate (i.e., the ablation rate due to 
material removal) and $q \square$ is the rate of energy density input from the laser beam, which is essentially a laser-induced body heat flux, and expressed as:

$$
\dot{q}=\alpha\left(1-R_{f}\right) I_{0}(t) e^{-\alpha z^{\prime}},
$$

where $\alpha$ and $R_{f}$ are, respectively, the absorption coefficient and the reflectivity, $z$ ' is the vertical distance from any points to the surface of the target material (i.e., $z^{\prime}=z$ - $d$, with $d$ is the corresponding ablation depth), and $I_{0}$ is the instantaneous laser irradiance at time $t$. Specifically, the temporal profile of the laser irradiance for the Nd:YAG laser pulse is assumed to follow the form proposed by Refs. [20, 22]:

$$
I_{0}(t)=I_{\max }\left(\frac{t}{t_{\max }}\right)^{7} \exp \left[7\left(1-\frac{t}{t_{\max }}\right)\right],
$$

where $I_{\max }$ is the peak irradiance of the laser pulse and $t_{\max }$ is the time when the laser irradiance reaches to its peak value.

The ablation rate $s \square$ in Eq. (1) represents the rate of material removal during the PLA process. In particular, under low laser fluence conditions (i.e., without considering material phase explosion), the material removal is predominantly attributed to evaporation, where the corresponding ablation rate $s \square$ can be described by using the Hertz-Knudsen equation [6]: 


$$
\dot{s}=\beta \sqrt{\frac{m}{2 \pi k_{B} T}} \frac{P_{b}}{\rho} \exp \left[\frac{m L_{v}}{k_{B}}\left(\frac{1}{T_{b}}-\frac{1}{T}\right)\right],
$$

where $\beta$ is the vaporization coefficient, $m$ is the atomic mass of the target solid material, $k_{B}$ is the Boltzmann constant, $L_{v}$ is the latent heat of vaporization of the material, and $T_{b}$ is the boiling temperature at the pressure $P_{b}$, and $P_{b}=1.01 \times 10^{5} \mathrm{~Pa}[23,24]$.

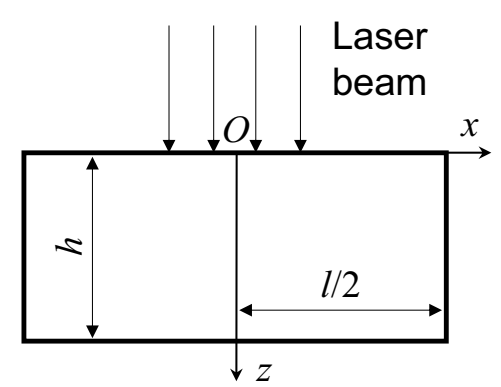

(a)

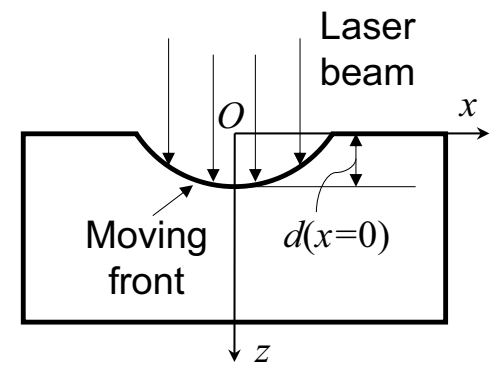

(b)

Fig. 1. Laser-material interaction considering moving front due to material removal: (a) at the beginning of laser ablation, and (b) during laser ablation.

Note that, in this paper, the ablation depth due to the material phase explosion, under high laser fluence conditions including plasma formation, is not considered. Moreover, the initial and boundary conditions for the current $2 \mathrm{D}$ problem are:

$$
\left.T(x, z, t)\right|_{t=0}=T_{a},
$$




$$
\begin{gathered}
\left.\frac{\partial T(x, z, t)}{\partial x}\right|_{x=-l / 2}=\left.\frac{\partial T(x, z, t)}{\partial z}\right|_{z=h}=0, \\
\left.k \frac{\partial T(x, z, t)}{\partial z}\right|_{z=0}=L_{V} \rho \dot{s},
\end{gathered}
$$

where $T_{a}$ is the room temperature, i.e., $T_{a}=300 \mathrm{~K}, l$ and $h$ are, respectively, the length and thickness of the target domain. Equation (6) represents the adiabatic boundary conditions on both the vertical side and the bottom surfaces, while Eq. (7) represents the heat loss on the top surface of the target material due to vaporization. Such boundary conditions have also been used by Refs. $[1,20,21]$. Note that the surface radiation to the ambient boundary condition is ignored due to the extreme short duration of the laser pulse. The heat loss due to surface radiation was found to be insignificant, when compared to the heat loss due to material vaporization and heat conduction (see also Ref. [21] for additional discussions).

In what follows, we assume that the uncertainty is associated with absorption coefficient and we provide the $\mathrm{gPC}$ representation for the governing equation for the laser heat conduction (see Eq. (1)), in terms of a single random variable $\xi, \alpha=\sum_{i=0}^{P} \alpha_{i} \psi_{i}$, where $\psi_{i}$ denotes the Legendre polynomial basis functions of order $i$. The first four Legendre polynomials are: $\psi_{0}(\xi)=1, \psi_{1}(\xi)=\xi, \psi_{2}(\xi)=\left(3 \xi^{2}-1\right) / 2$, and $\psi_{3}(\xi)=\left(5 \xi^{3}-3 \xi\right) / 2$ (see Ref. [14] for more details regarding Legendre polynomial basis functions), where $\xi$ denotes all conceivable values within the lower $\left(\xi_{\min }\right)$ and upper bounds $\left(\xi_{\max }\right)$ of absorption coefficients at a certain temperature. Furthermore, $\alpha_{i}$ denotes the coefficients of Legendre polynomials, which represent 
the projections of absorption coefficients to different polynomial chaos modes. $\alpha_{i}$ can be obtained using:

$$
\alpha_{i}=\frac{2 \int_{\xi_{\min }}^{\xi_{\max }} \xi \psi_{i} d \xi}{\left(\xi_{\max }-\xi_{\min }\right)\left\|\psi_{i}\right\|^{2}},
$$

where $\left\|\psi_{i}\right\|^{2}$ is the normalization corresponding to the Legendre polynomial of order $i$ and is given by $\left\|\psi_{i}\right\|^{2}=\frac{2}{2 i+1}$. Here, the integral in Eq. (8) is calculated using the trapezoidal rule (the numerical error has found to be trivial). $P+1$ denotes the total number of PC mode strengths, and $P+1=(n+p) !=n ! p !$, where $p$ and $n$ are the truncated order and dimension of the PC expansion, respectively (e.g., see Refs. [10, 12-15] and the references therein for additional discussions). In this study, we truncated the PC expansion to the order of three (i.e., $p=3$, see Section 4.1 for detailed discussions). In addition, the stochastic dimension is $n=1$, since we only considered uncertainty in the absorption coefficient parameter.

Likewise, the associated gPC expansions, with finite variances, for the temperature and the surface recession rate are, respectively, $T=\sum_{i=0}^{P} T_{i} \psi_{i}$ and $\dot{s}=\sum_{i=0}^{P} \dot{s}_{i} \psi_{i}$, where $T_{i}$ and $\dot{s}_{i}$ are PC mode strengths of temperature and recession rate. Substituting these expansions into the governing equation of Eq. (1) results in: 


$$
\begin{aligned}
\rho C_{p} & \left(\sum_{i=0}^{P} \psi_{i} \frac{\partial T_{i}}{\partial t}-\sum_{i=0}^{P} \sum_{j=0}^{P} \psi_{i} \psi_{j} \dot{s}_{i} \frac{\partial T_{j}}{\partial z}\right)-\sum_{i=0}^{P} \psi_{i} \nabla \cdot\left(k \nabla T_{i}\right) \\
& =\left(1-R_{f}\right) I_{0}(t) \sum_{i=0}^{P} \alpha_{i} \psi_{i} \exp \left(-z^{\prime} \sum_{i=0}^{P} \alpha_{i} \psi_{i}\right) .
\end{aligned}
$$

Further, applying the Galerkin projection, which involves multiplying Eq. (9) by $\psi_{k}$, and then evaluating the expectation, making use of orthogonality, and finally dividing by $\left\langle\psi_{k}^{2}\right\rangle$, for $\mathrm{k}$ $=0,1, \ldots, P$, we get the following coupled system equations (i.e., a set of coupled partial differential equations)

$$
\begin{aligned}
\rho C_{p} & \frac{\partial T_{k}}{\partial t}-\rho C_{p} \sum_{i=0}^{P} \sum_{j=0}^{P} \dot{s}_{i} \frac{\partial T_{j}}{\partial z} C_{i j k}-\nabla \cdot\left(k \nabla T_{k}\right) \\
= & \frac{\left(1-R_{f}\right) I_{0}(t)}{\left\langle\psi_{k}^{2}\right\rangle}\left\langle\sum_{i=0}^{P} \alpha_{i} \psi_{i} \exp \left(-z^{\prime} \sum_{i=0}^{P} \alpha_{i} \psi_{i}\right), \psi_{k}\right\rangle,
\end{aligned}
$$

where the tensor $C_{i j k}$ is a known property of the basis, which can be computed as follows

$$
C_{i j k}=\frac{\left\langle\psi_{i} \psi_{j} \psi_{k}\right\rangle}{\left\langle\psi_{k}^{2}\right\rangle}, \quad i, j, k \in\{0,1, \ldots, P\},
$$


This allows an easy computation of the PC expansion of any two-term products. Note that a similar treatment for the initial and boundary conditions in Eqs. (5)-(7) also gives the following boundary conditions

$$
\begin{gathered}
\left.T_{0}(x, z, t)\right|_{t=0}=\left.\frac{1}{\left\langle\psi_{0}^{2}\right\rangle} T_{a} \quad \& \quad T_{k}(x, z, t)\right|_{t=0}=0, \quad k=1, \ldots, P, \\
\left.\frac{\partial T_{k}(x, z, t)}{\partial x}\right|_{x=l / 2}=\left.\frac{\partial T_{k}(x, z, t)}{\partial z}\right|_{z=h}=0, \quad k=0,1, \ldots, P \\
\left.k \frac{\partial T_{k}(x, z, t)}{\partial z}\right|_{z=0}=L_{v} \rho \dot{s}_{k}, \quad k=0,1, \ldots, P .
\end{gathered}
$$

Here, we remark that we use a fractional step projection scheme to integrate numerically the coupled system equations in Eq. (10) along with the initial and boundary conditions of Eqs. (12)-(14).

\section{Material Properties and Uncertain Temperature-dependent Absorption Coefficient}

In this paper, the target material used for the current study is pure aluminum. The following material properties of the aluminum target are assumed to be deterministic: the density, thermal conductivity, specific heat, melting and boiling temperature, boiling pressure, vaporization coefficient, latent heat of vaporization, critical temperature, and critical density (uncertainties could also exist in these material parameters, quantifying such uncertainties will be our future work). In addition, the density, thermal conductivity, specific heat are also 
temperature-dependent. The values for these deterministic material properties are collected from various sources [20, 23-27] and tabulated in Table 1.

The laser source considered in this study is an Nd:YAG pulsed laser with wavelength of $266 \mathrm{~nm}$. The total pulse duration is $6 \mathrm{~ns}$. The temporal variation of the laser pulse follows Eq. (3) with a full-width half max of 2.24 ns. The laser beam is a Gaussian beam with a spatial profile of $\dot{q} \cdot \exp \left(-7.6 x^{2} / r^{2}\right)$, where $r$ is the radius of the laser beam, $r=50 \mu \mathrm{m}$. Such a Gaussian profile is chosen by fitting the spatial profile of the laser beam determined from experimental tests reported by Ref. [28]. The absorption coefficient and reflectivity of aluminum are both temperature and wavelength-dependent. Meanwhile, the reflectivity is assumed to be deterministic, while the absorption coefficient is uncertain. The absorption coefficient values reported by Refs. [20, 21, 23, 24, 26, 29, 30] are collected, and are allowed to vary over a range that covers all conceivable values, i.e., $\xi \in U\left(\xi_{\min }, \xi_{\max }\right)$. Table 2 lists the obtained lower $\left(\xi_{\min }\right)$ and upper bounds $\left(\xi_{\max }\right)$ of the temperature-dependent absorption coefficient and the deterministic temperature-dependent reflectivity. Note that the variability in the absorption coefficient values are passed into the gPC representations as uniformly distributed random variables, denoted by $U(a, b)$. In addition, we used a stratified sampling technique for which the range for the uncertain absorption coefficient parameter is divided into segments of equal probability (i.e., $\xi\left(\xi_{\min }, \xi_{\min }+\frac{\xi_{\max }-\xi_{\min }}{99}, \xi_{\min }+\frac{2\left(\xi_{\max }-\xi_{\min }\right)}{99}, \ldots, \xi_{\max }\right)$ ). Note that, before passing the sample values from these intervals into the Legendre polynomials, $\psi_{i}(\xi)$ (see Eq. 9 and paragraph above), the samples are mapped into an appropriate range of $[-1,1]$ (i.e., the applicable range for the Legendre polynomials). Also note that increasing the number of sample 
values within the lower and upper bounds may improve the accuracy of the mean prediction of the output; however, the computational effort will substantially increase with the number of sample sizes.

Table 1. Literature based material properties of aluminum used in the simulation studies.

\begin{tabular}{|c|c|c|c|}
\hline \multicolumn{2}{|c|}{ Material property } & Temperature & Unit \\
\hline Melting temperature & $T_{m}=933$ & & K \\
\hline Critical temperature & $T_{c}=8860$ & & $\mathrm{~K}$ \\
\hline Critical density & $\rho_{c}=300$ & & $\mathrm{~kg} / \mathrm{m}^{3}$ \\
\hline \multirow{2}{*}{ Density } & $\rho=2700$ & $T \leq T_{m}$ & $\mathrm{~kg} / \mathrm{m}^{3}$ \\
\hline & $\rho=\rho_{c}\left(1+0.75\left(1-T / T_{c}\right)+3\left(1-T / T_{c}\right)^{1 / 3}\right)$ & $T_{m}<T \leq T_{c}$ & $\mathrm{~kg} / \mathrm{m}^{3}$ \\
\hline \multirow{2}{*}{ Specific heat } & $C_{p}=0.5203 T+643.9$ & $T \leq T_{m}$ & $\mathrm{~J} /(\mathrm{kg} \cdot \mathrm{K})$ \\
\hline & $C_{p}=1160$ & $T_{m}<T \leq T_{c}$ & $\mathrm{~J} /(\mathrm{kg} \cdot \mathrm{K})$ \\
\hline Thermal conductivity & $k \in(16,237)[26]$ & $T_{a}<T \leq T_{c}$ & $\mathrm{~W} /(\mathrm{m} \cdot \mathrm{K})$ \\
\hline Boiling temperature & $T_{b}=2743$ & & $\mathrm{~K}$ \\
\hline Boiling pressure & $P_{b}=1.01 \times 10^{5}$ & & $\mathrm{~Pa}$ \\
\hline Vaporization coefficient & $\beta=0.82$ & & \\
\hline $\begin{array}{l}\text { Latent heat of } \\
\text { vaporization }\end{array}$ & $L_{v}=10.78$ & & $\mathrm{MJ} / \mathrm{kg}$ \\
\hline
\end{tabular}

Table 2. Temperature-dependent absorption coefficients (lower and upper bounds) and temperature-dependent reflectivity of aluminum under the $266 \mathrm{~nm}$ wavelength laser.

\begin{tabular}{|c|c|c|c|}
\hline \multirow{2}{*}{ Temperature, $\mathrm{K}$} & \multicolumn{2}{|c|}{ Absorption coefficient, 1/m } & \multirow{2}{*}{ Reflectivity } \\
\cline { 2 - 3 } & Lower bound, $\xi_{\min }$ & Upper bound, $\xi_{\max }$ & \\
\hline 300 & $3.83 \times 10^{8}$ & $4.50 \times 10^{8}$ & $80.00 \%$ \\
\hline 600 & $3.83 \times 10^{8}$ & $4.50 \times 10^{8}$ & $78.67 \%$ \\
\hline 933 & $3.83 \times 10^{8}$ & $4.50 \times 10^{8}$ & $77.20 \%$ \\
\hline
\end{tabular}




\begin{tabular}{|l|l|l|l|}
\hline 2000 & $3.55 \times 10^{8}$ & $4.17 \times 10^{8}$ & $72.47 \%$ \\
\hline 4000 & $3.01 \times 10^{8}$ & $3.54 \times 10^{8}$ & $63.61 \%$ \\
\hline 6031 & $2.47 \times 10^{8}$ & $2.90 \times 10^{8}$ & $54.75 \%$ \\
\hline 7000 & $1.64 \times 10^{8}$ & $1.92 \times 10^{8}$ & $50.32 \%$ \\
\hline 8000 & $7.78 \times 10^{7}$ & $9.13 \times 10^{7}$ & $45.88 \%$ \\
\hline 8860 & $3.83 \times 10^{6}$ & $4.50 \times 10^{6}$ & $42.07 \%$ \\
\hline
\end{tabular}

\section{Computational Implementation and Sensitivity Analysis Approach}

\subsection{A finite element analysis (FEA) framework that accounts for the absorption coefficient uncertainties}

A finite element analysis (FEA) for the laser heat conduction problem was implemented using ABAQUS with user subroutines including the DFLUX and the UMESHMOTION subroutines [31], as well as additional in-house developed utility subroutines, written in FORTRAN. In particular, the DFLUX subroutine is used to define the heat flux of the laser source in Eq. (2), while the UMESHMOTION subroutine defines the recession rate of the surface material removal in Eq. (4), which enables the coupling between the laser heat conduction and the progressive material removal. Such a computational framework has been used in our recent work [8] on modeling the PLA of aluminum, but without considering any uncertainties in the material parameters. Moreover, the in-house developed utility subroutines are used to implement the gPC expansions within the representation of the laser heat conduction phenomena (i.e., Eq. (10)). Here, the computational domain of the problem is $150 \mu \mathrm{m}$ long $(x$ direction) and $10 \mu \mathrm{m}$ thick (z-direction), which represents the entire region of the target material (see also Fig. 1). The domain is meshed with ABAQUS CPE4T elements (i.e., the 4-node plane strain coupled temperature-displacement elements). To balance the accuracy and computational 
cost, the meshes at the region near the heated surface are refined (with mesh size of $0.8 \mu \mathrm{m}$ by $0.05 \mu \mathrm{m}$ ) and a coarse mesh (with mesh size of $0.8 \mu \mathrm{m}$ by $0.5 \mu \mathrm{m}$ ) is used for the region far away from the heated surface. The meshing results in a total of 15980 elements. Moreover, the automatic time incrementation algorithm is enabled in ABAQUS. The minimum and maximum time increments are $0.1 \mathrm{fs}$ and $0.1 \mathrm{~ns}$, respectively. The duration of the laser heat conduction step is $6 \mathrm{~ns}$. In addition, the laser heat conduction step is followed by a cool down step with a duration of $200 \mathrm{~ns}$, in which the laser-induced body heat flux is removed from the computation and only the conduction of the remaining heat within the target material is considered. Note that the surface temperature of the target material right at the end of laser pulse is usually above the boiling point, which suggests the continuation of ablation according to Eq. (4). Therefore, adding a cool down step after the end of the laser pulse allows us to capture the continuation of ablation and the temperature drop. During the implementation, ABAQUS automatically adjusts the time step to ensure the numerical stability and convergence. The selected mesh size and the automatic time incrementation scheme provide sufficient numerical accuracy based on our preliminary mesh refinement studies. Similar to ensuring convergence of the mesh resolution, the number of Legendre polynomial basis functions used in the gPC expansion was increased until the truncation error of the gPC expansion becomes negligible. This was achieved by computing the difference between a lower order and a higher order gPC expansion. When the maximum pointwise difference between the lower order and that of the higher order gPC expansions was very small (i.e., a relative ratio less than $1.1 \times 10^{-4}$ ) for the predicted temperature or ablation response, then the gPC expansion was assumed converged. Using the user defined software along with ABAQUS, the gPC expansion order is inferred from the quadrature order in stochastic space. A quadrature order of three was found to achieve truncation error convergence in the gPC 
expansions (i.e., $p=3$, see Section 2). Furthermore, the simulations were carried out under three laser fluence conditions, i.e., $13.73,17.70$, and $22.68 \mathrm{~J} / \mathrm{cm}^{2}$. Note that these are low laser fluence conditions (i.e., without material phase explosion) which correspond to pulse energies between 1 and $2 \mathrm{~mJ}$.

\subsection{Sensitivity analysis approach}

Sensitivity analysis is also performed in this study. The approach of sensitivity analysis taken here is similar to that of the variance-based sensitivity analysis proposed by Ref. [32], where such a sensitivity analysis procedure provides information with respect to output dispersion measures such as variance. In particular, in the variance-based case, the goal is to apportion the variance of the quantity of interest among its various factor contributors and seek to identify the most significant factor contributors providing the most variance in the temperature and/or the ablation response. Here the sensitivity study was performed by investigating the effect of uncertainty ranges with respect to the upper and lower values of the absorption coefficient on the mean predictions of the temperature and ablation response.

\section{Results and Discussions}

\subsection{Effects of absorption coefficient uncertainties on the temperature and ablation response}

The formulation described in Section 2 (i.e., Eq. (10) with boundary conditions of Eqs. (12)-(14)) is implemented using the proposed FEA framework described in Section 4.1. The input parameters include thermal conductivity, specific heat, density, reflectivity, absorption 
coefficient, and etc. In this study, we only considered uncertainties in temperature-dependent absorption coefficients (see Table 2), while the other parameters are assumed to be deterministic (see Table 1). For a nanosecond Nd: YAG pulsed laser, with wavelength $266 \mathrm{~nm}$, fluence 13.73 $\mathrm{J} / \mathrm{cm}^{2}$ and duration $6 \mathrm{~ns}$, the predicted temperature histories in the center (i.e., $x=0$ and $z^{\prime}=0$ ) of the aluminum target surface corresponding to four PC mode strengths (i.e., $T_{0}, T_{1}, T_{2}$ and $T_{3}$ temperature fields) are shown in Fig. 2. Note that the temperature history corresponding to the first PC mode $T_{0}$, which is the mean prediction for the temperature histories due to uncertainty in the absorption coefficient, shows that the temperature rapidly increases to an upper limit of approximately $8700 \mathrm{~K}$ within $1 \mathrm{~ns}$. Moreover, the temperature history for the second PC mode $T_{1}$ shows a similar temperature trend with the same order as that of the first PC mode $T_{0}$. However, the temperature histories for the third and fourth PC modes (i.e., $T_{2}$ and $T_{3}$ temperature fields) clearly show that temperature values are two orders lower than that of the first and second PC modes, which further shows that the truncation error convergence in the gPC expansions can be achieved with quadrature order of three. 


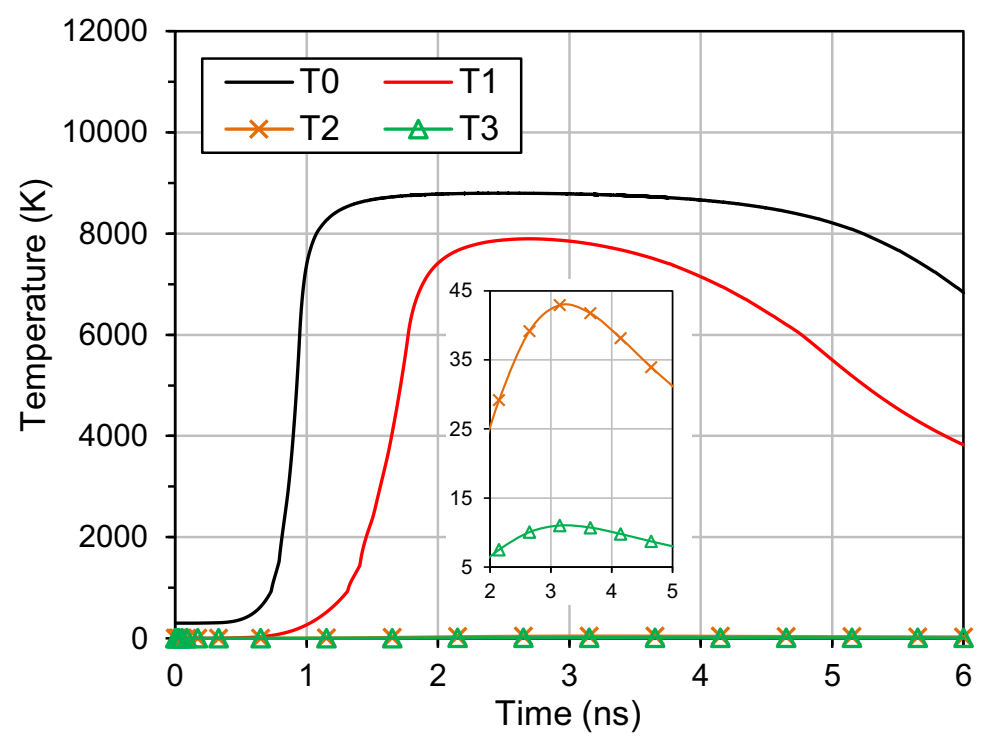

Fig. 2. The polynomial chaos mode strengths of the temperature histories, $T_{0}, T_{1}, T_{2}$ and $T_{3}$, in the center of the top surface of the aluminum target (i.e., $x=0$ and $z^{\prime}=0$ ) under laser fluence of

$$
13.73 \mathrm{~J} / \mathrm{cm}^{2} \text {. }
$$

To further illustrate the thermal response, the temperature distributions in the center region of the aluminum target (i.e., $-0.4 \mu \mathrm{m} \leq x \leq 0.4 \mu \mathrm{m}$, where one element is used in the $x$ direction, see Fig. 1) and at the end of the PLA step (i.e., when $t=6 \mathrm{~ns}$ ) are shown in Fig. 3. In particular, Figs. 3(a)-(d) show the predicted temperature fields corresponding to those four PC modes, i.e., $T_{0}, T_{1}, T_{2}$ and $T_{3}$ temperature fields, respectively, whereas Fig. 3(e) shows the standard deviation $\sigma_{T}=\sqrt{\sum_{k=1}^{3} T_{k}^{2}\left\langle\psi_{k}^{2}\right\rangle}$ of the predicted temperature fields (i.e., the projected standard deviation on the un-deformed geometry) due to the uncertainty in the absorption coefficient. Note that the predicted temperature values of the third and fourth PC modes are significantly lower than that of the first and second PC modes, i.e., similar observations to those results in Fig. 2. In addition, the mean predictions for the temperature history in the center (i.e., 
$x=0$ and $z^{\prime}=0$ ) of the aluminum target under the three laser fluence, $13.73,17.70$ and $22.68 \mathrm{~J} / \mathrm{cm}^{2}$, are compared in Fig. 4. As one can see, the temperatures under those three laser fluences all reach to an upper limit of around $8700 \mathrm{~K}$. Such an upper temperature is physically defined when a critical temperature is used. However, the higher the fluence, the faster the temperature grows, and the earlier the upper temperature is reached. Note that the increase of the laser fluence leads to an increase of the laser energy input (see Eq. (2)) and, thus, a more rapid temperature rise in the target material. Meanwhile, the ablation rate also increases rapidly with the temperature rise (see Eq. (4)). As a result, the material with high temperature progressively ablates which counterbalances the temperature increase. For the cases when the rate of the temperature rise is much larger than the ablation rate (e.g., fluence $>22.68 \mathrm{~J} / \mathrm{cm}^{2}$ ), the thickness of the region where the temperature is above $0.9 T_{c}$ can continue to increase. If such a thickness exceeds a certain limit (e.g., the critical diameter of vapor bubbles), material phase explosion can occur [9]. Note that such cases are not considered in this study. In addition, the energy balance of the center region of the target material (i.e., $-0.4 \mu \mathrm{m} \leq x \leq 0.4 \mu \mathrm{m}$, see Figs. 1 and 3) during the PLA process is roughly estimated. The energy loss due to the mass removal is calculated by adding the sensitive heat (i.e., $m C_{p} \Delta T$, where $m$ is the ablated mass and $\Delta T$ is the temperature change) to the phase change energy $\left(m L_{v}\right)$. Then, the energy conducted away within the remaining target material is the difference between the total pulse energy and the energy loss due to the mass removal. Figure 5 shows the comparison of the total pulse energy, the energy loss attributed to the ablated mass, and the energy conducted away within the remaining target material under the three laser fluence conditions. 


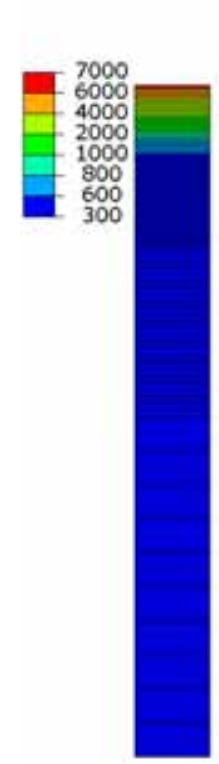

(a)

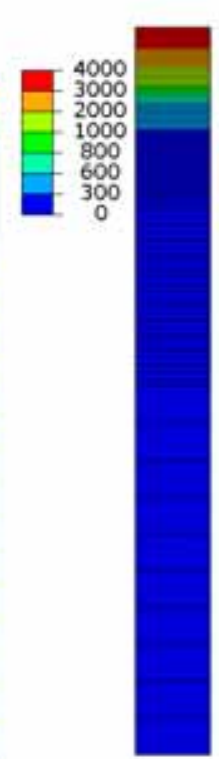

(b)

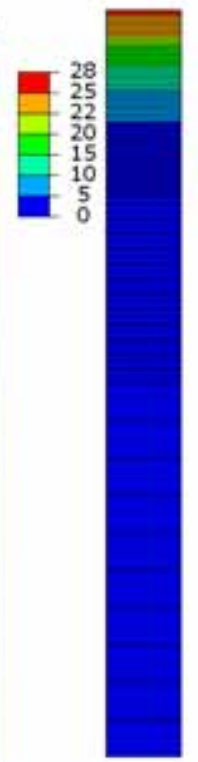

(c)

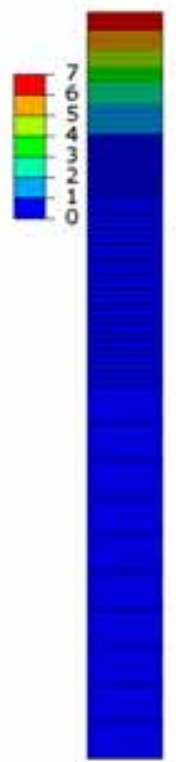

(d)

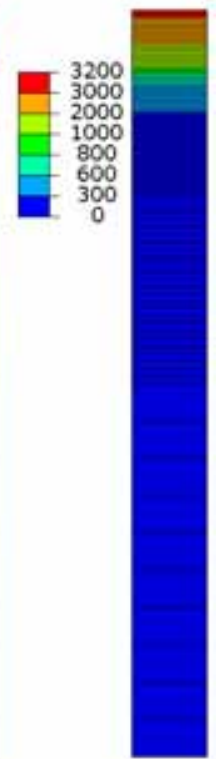

(e)

Fig. 3. The polynomial chaos mode strengths of the temperature distributions at the end of the PLA step (i.e., at $t=6 \mathrm{~ns}$ ), (a) $T_{0}$, (b) $T_{1}$, (c) $T_{2}$ and (d) $T_{3}$, as well as (e) the standard deviation $\sigma_{T}$ of the temperature, under laser fluence of $13.73 \mathrm{~J} / \mathrm{cm}^{2}$.

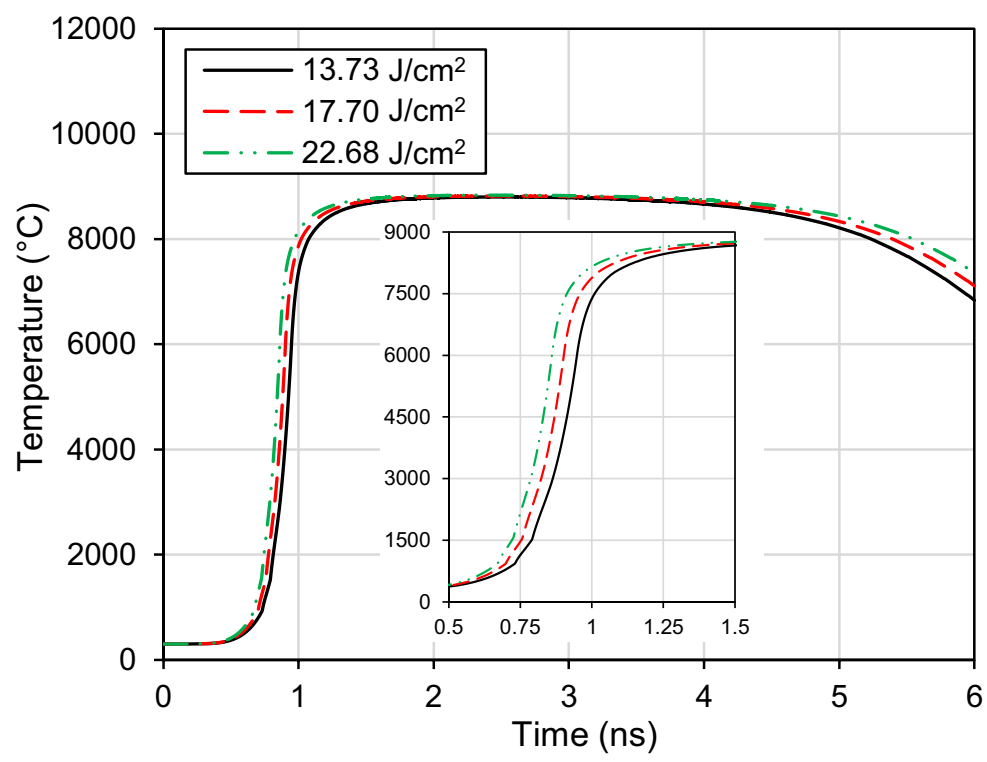

Fig. 4. The mean predictions for the temperature history in the center (i.e., $x=0$ and $z^{\prime}=0$ ) of the aluminum target under the three laser fluence conditions, $13.73,17.70$ and $22.68 \mathrm{~J} / \mathrm{cm}^{2}$. 


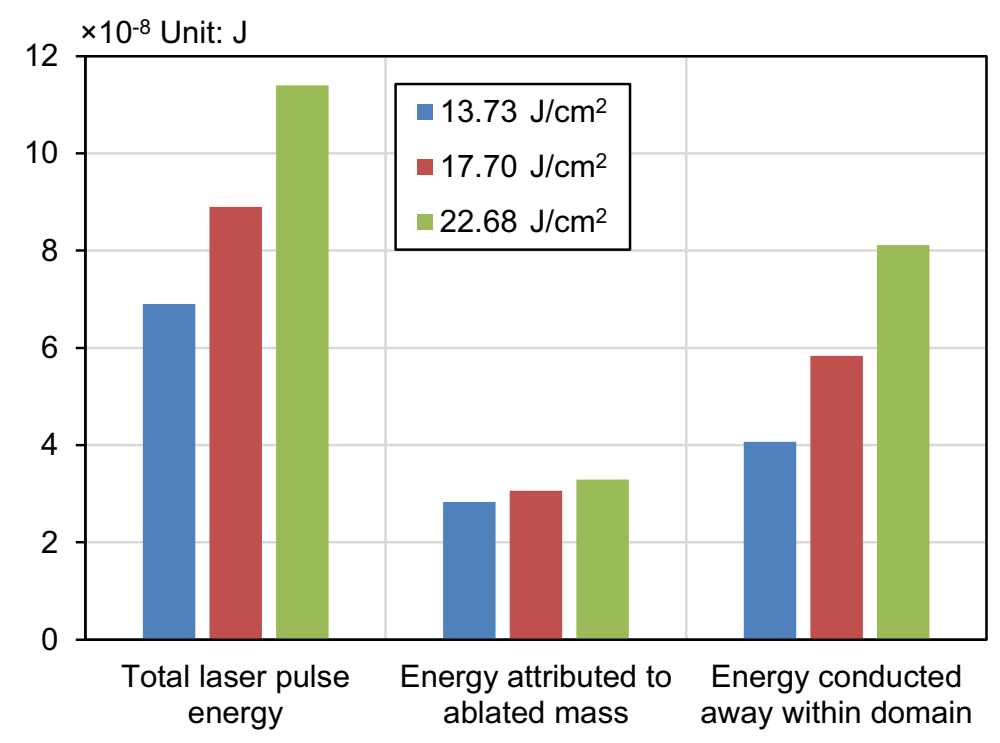

Fig. 5. Total laser pulse energy, energy attributed to the ablated mass, and energy conducted away within the remaining aluminum target (for the element portions shown in Fig. 3) under the three laser fluence conditions, $13.73,17.70$ and $22.68 \mathrm{~J} / \mathrm{cm}^{2}$.

Moreover, the predicted ablation depths after the laser pulse in the center of the aluminum target surface corresponding to those four PC modes (i.e., $U_{0}, U_{1}, U_{2}$ and $U_{3}$ ) under fluence of $13.73,17.70$, and $22.68 \mathrm{~J} / \mathrm{cm}^{2}$, are shown in Fig. 6 . The corresponding mean predictions for the ablation depth due to uncertainty in the absorption coefficient are 1.011, 1.092, and $1.173 \mu \mathrm{m}$, respectively. Here, it can be seen that the predicted ablation depths under the third and fourth PC modes, i.e., $U_{2}$ and $U_{3}$, are almost zero, which is not surprising as the predicted temperatures under those two modes (see also Figs. 2 and 3) are too low to cause material ablation. It is also worth mentioning that the ablation depth results presented throughout this study are predicted at $200 \mathrm{~ns}$ after the end of the laser pulse. Figure 7 shows the temperature rapidly drops below the boiling point within $7.5 \mathrm{~ns}$ and the ablation depth extends from 1.0076 
$\mu \mathrm{m}$ to $1.0115 \mu \mathrm{m}$ shortly after the end of the laser pulse (under the laser fluence condition of $13.73 \mathrm{~J} / \mathrm{cm}^{2}$ ). Therefore, without considering the continuation of ablation after the end of laser pulse can slightly underestimate the total ablation depth. In addition, Fig. 8 shows the comparisons of the mean predictions of the ablation depth under three laser fluence conditions with the experimental data that are reported by Ref. [5] and the predictions using the hydrodynamics method reported by Ref. [2]. As one can see, the mean predictions obtained from our simulations compare favorably with the experimental data, with the percentage errors being $1.2 \%, 3.0 \%$, and $9.1 \%$, respectively, under three laser fluence conditions. Here, we remark that the predictions using the hydrodynamics method with deterministic material parameters underestimated the ablation depths when compared to the experimental data.

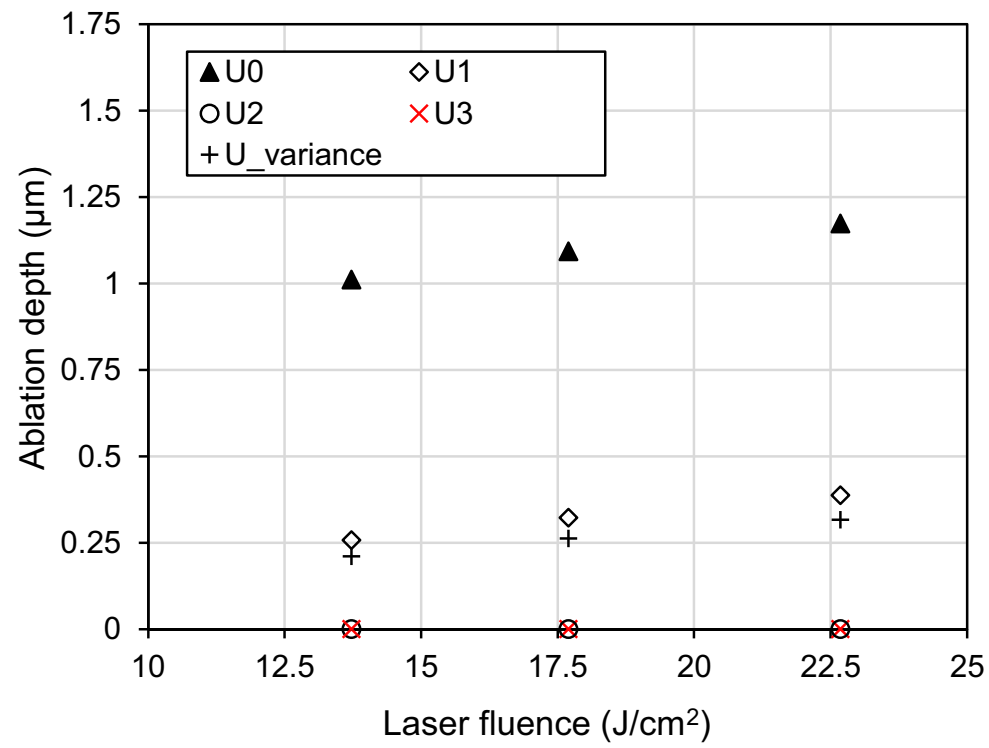

Fig. 6. The polynomial chaos mode strengths of the ablation depths after the laser pulse under the three laser fluence conditions, $13.73,17.70$ and $22.68 \mathrm{~J} / \mathrm{cm}^{2}$. 


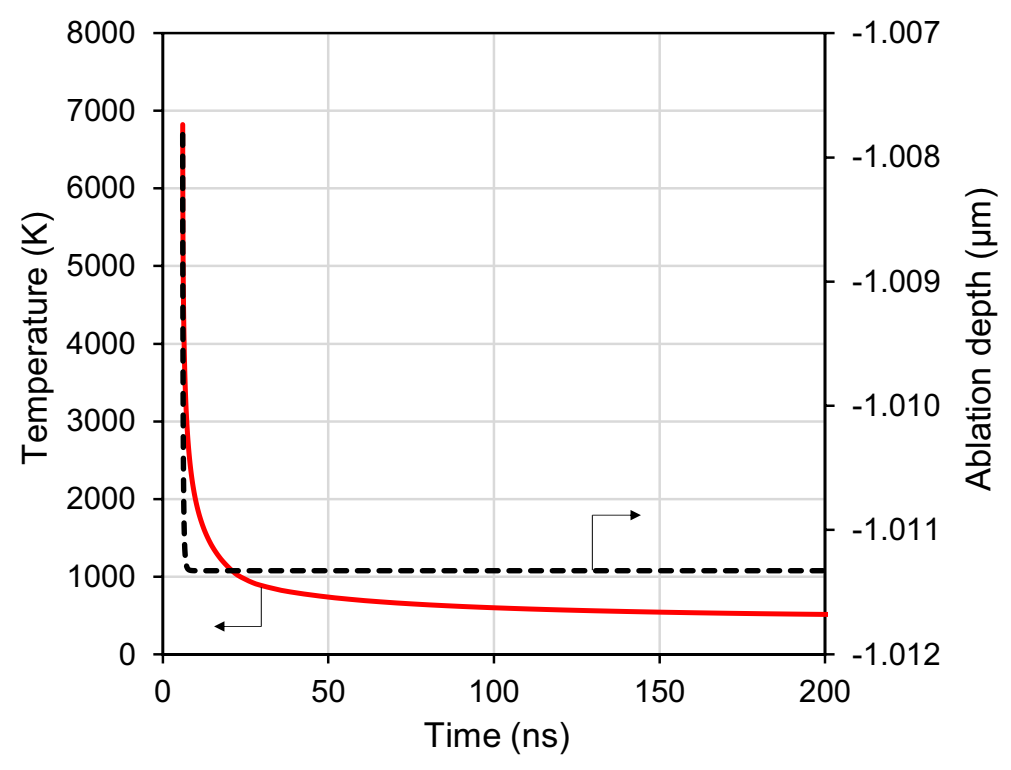

Fig. 7. Temperature drop and the continuation of ablation after the end of the laser pulse under the laser fluence condition of $13.73 \mathrm{~J} / \mathrm{cm}^{2}$.

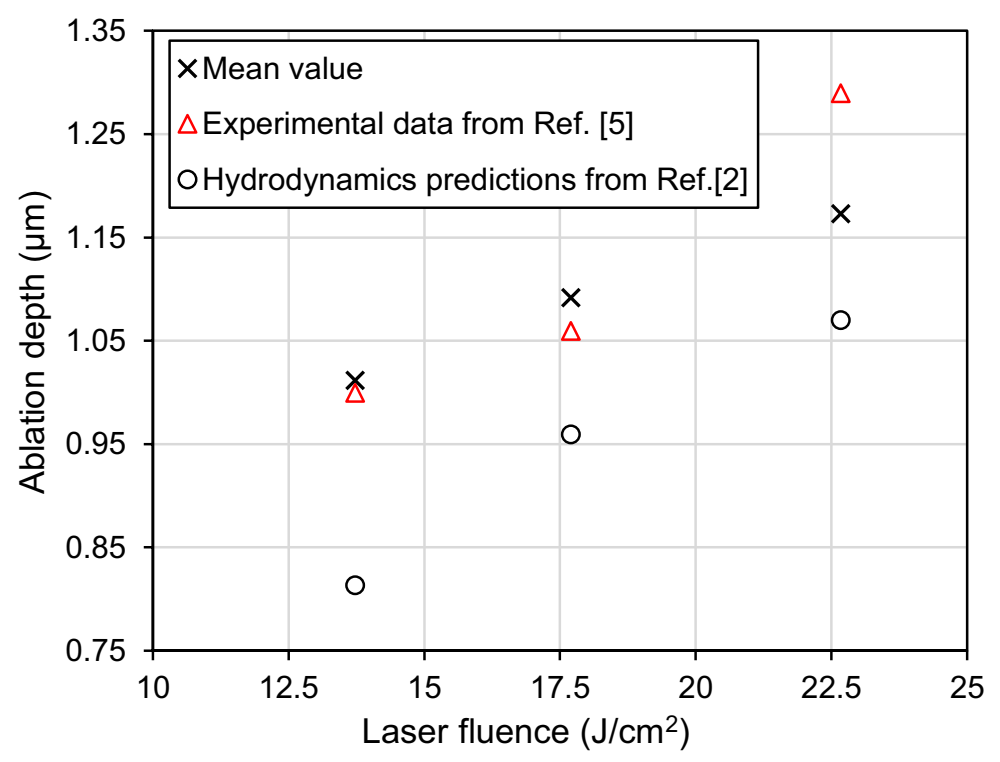

Fig. 8. Ablation depths after the laser pulse under three fluence levels: comparisons between mean predictions of ablation depths considering uncertainties within the temperature-dependent 
absorption coefficient, experimental data reported by Ref. [5], and predictions using hydrodynamics method with deterministic material parameters reported by Ref. [2].

Furthermore, Fig. 9 shows the contour plots of the mean temperature distributions in the target material at $t=3$ and $6 \mathrm{~ns}$, where the shape change of the target material is also captured.

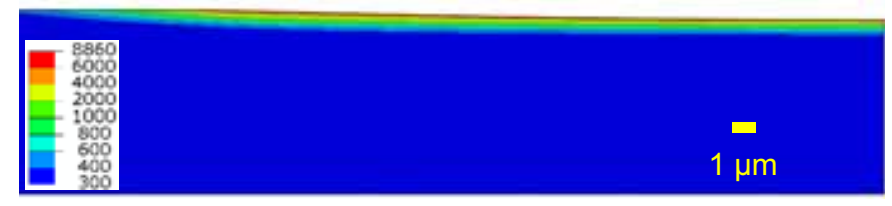

(a) $t=3$ ns

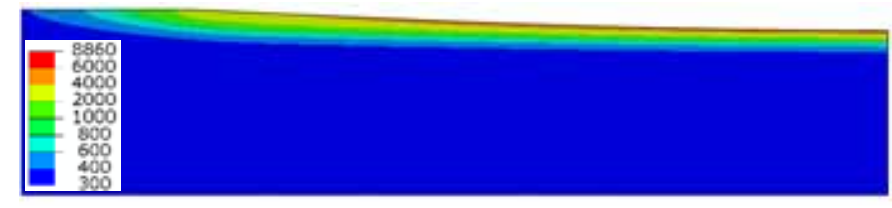

(b) $t=6$ ns

Fig. 9. Contour plots of mean temperature distributions in the left half (i.e., $x \leq 0$, see Fig. 1) of the aluminum target at $t=3$ and $6 \mathrm{~ns}$ under laser fluence of $22.68 \mathrm{~J} / \mathrm{cm}^{2}$.

\subsection{Results of sensitivity analysis}

The sensitivity analysis performed in this study is an extension of our investigation on effects of the uncertain absorption coefficients on the temperature and ablation response. Such an analysis provides us understandings on how the changes of the uncertainty range of the absorption coefficient can influence the mean predictions of the temperature and ablation 
response. The results from the sensitivity analysis could also be used in inverse problems to narrow down the range of the true absorption coefficient of the target material.

In this study, sensitivity studies for each laser fluence condition were performed, when the lower and/or upper bound of the absorption coefficient is varied by a $10 \%$ perturbation (i.e., decreasing the minimum and/or maximum values of the absorption coefficients by $10 \%$ ). The mean ablation depths predicted from these study cases are then compared to the mean ablation depth predicted from the case without perturbation for each laser fluence level, as shown in Fig. 10. Notice that the mean ablation depths predicted from the cases with perturbations are slightly lower than those predicted from the cases without perturbation.

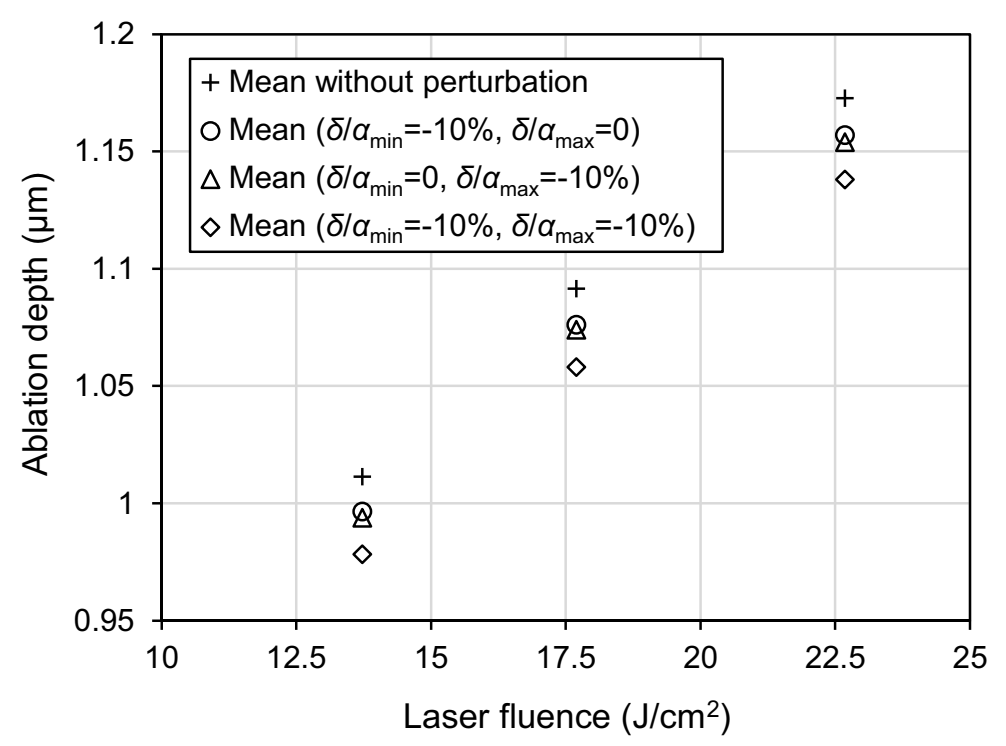

Fig. 10. Ablation depths after the laser pulse: comparisons between the predicted mean value with no perturbation and predicted mean values when a $10 \%$ perturbation is applied to decrease the minimum values of absorption coefficient (i.e., $\delta / \alpha_{\min }=-10 \%$ ), the maximum values of the 
absorption coefficient (i.e., $\delta / \alpha_{\max }=-10 \%$ ), and both the minimum and maximum values of the absorption coefficient (i.e., $\delta / \alpha_{\min }=\delta / \alpha_{\max }=-10 \%$ ).

Furthermore, additional simulation cases with $-5 \%, 5 \%$, and $10 \%$ perturbations of the minimum or maximum absorption coefficients were performed (with laser fluence $13.73 \mathrm{~J} / \mathrm{cm}^{2}$ ). The predicted mean ablation depths from all additional simulation cases with and without perturbations are compared in Fig. 11. The comparison shows that decreasing either the minimum or maximum values of the absorption coefficients leads to an almost linear decrease to the predicted mean ablation depths, while increasing those values leads to an almost linear increase to the predicted mean ablation depths. In particular, a 10\% change in either the lower or upper bound of the absorption coefficient can lead to an approximate $1.8 \%$ change in the predicted mean ablation depths. In addition, the predicted mean ablation depth shows good agreement with the experiment data when either the minimum or maximum values of the absorption coefficients are decreased by $5 \%$. However, the difference between the predicted mean ablation depth and the experimental data increases when the perturbation is decreased from $-5 \%$ to $-10 \%$ (see also Fig. 11). This finding can be used to locate the approximate true value of the absorption coefficient. For the current case, the true value of the absorption coefficient is approximately located within the range between $95 \%$ of the lower bound and $95 \%$ of the upper bound, but excluding the range between $90 \%$ of the lower bound and $90 \%$ of the upper bound. 


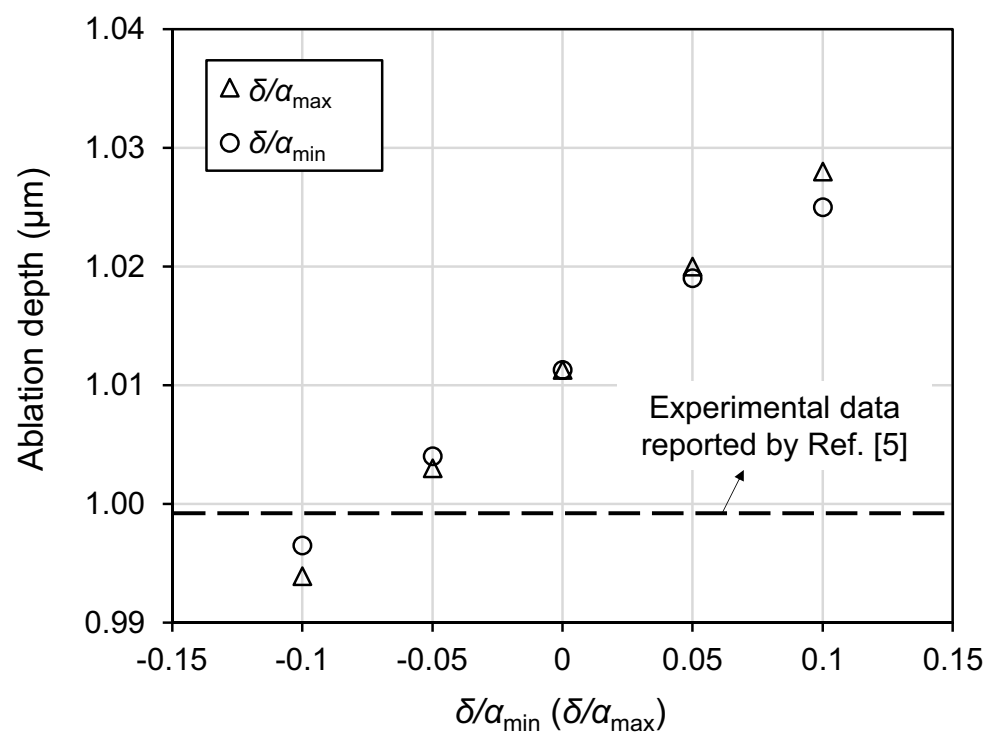

Fig. 11. Ablation depths after the laser pulse: comparisons of predicted mean values with no perturbation, predicted mean values when $-10 \%,-5 \%, 5 \%$ and $10 \%$ perturbations are applied to the minimum and maximum values of the absorption coefficient, and experimental data [5], under laser fluence of $13.73 \mathrm{~J} / \mathrm{cm}^{2}$.

\section{Conclusion}

In this paper, a generalized polynomial chaos (gPC) based uncertainty quantification (UQ) for modeling pulsed laser ablation (PLA) of aluminum is considered. In particular, the gPC based UQ for PLA is used to incorporate the parameter uncertainty of the temperature-dependent absorption coefficient within the representation of laser heat conduction phenomena. The proposed gPC based UQ is implemented through numerical simulations with finite element analysis. Numerical simulation studies were presented for a nanosecond Nd:YAG $266 \mathrm{~nm}$ pulsed laser under different low laser fluence conditions (i.e., without considering the phase explosion and plasma formation). The simulations successfully captured four PC mode strengths, mean 
predictions, and standard deviations of the surface temperature and ablation histories of the target material considering the uncertainties in the temperature-dependent absorption coefficient. Results showed that the mean predictions of the ablation depths under the three laser fluence levels compare well with the experimental data (with the percentage errors being $1.2 \%, 3.0 \%$, and 9.1\%). Moreover, a sensitivity analysis was performed and the results showed that a $10 \%$ change in either the lower or upper bound of the absorption coefficient can lead to an approximate $1.8 \%$ change in the predicted mean ablation depths. Of particular interest of future

study would be to investigate the effects of uncertainties in two or more parameters (e.g., thermal conductivity, specific heat) and combining such an analysis with, albeit limited, experimental data for validation purpose within the representation of laser heat conduction phenomena.

\section{Acknowledgements}

This work was supported in part by the Air Force Research Laboratory (AFRL) under prime contract no. FA8651-08-D-0108 and task order no. 42. Any opinions, findings, conclusions, or recommendations expressed in this work are those of the authors and do not necessarily reflect the views of the AFRL. Y. Wang also thanks Dr. Crystal L. Pasiliao (AFRL/RW) for her support in this research and Dr. Ninggang Shen (General Motors) for many helpful discussions. 


\section{References}

[1] N.M. Bulgakova, A.V. Bulgakov, Pulsed laser ablation of solids: transition from normal vaporization to phase explosion, Applied Physics A: Materials Science \& Processing, 73(2) (2001) 199-208.

[2] Y. Cao, X. Zhao, Y.C. Shin, Analysis of nanosecond laser ablation of aluminum with and without phase explosion in air and water, Journal of Laser Applications, 25(3) (2013) 032002.

[3] G. Cristoforetti, S. Legnaioli, V. Palleschi, E. Tognoni, P.A. Benedetti, Observation of different mass removal regimes during the laser ablation of an aluminium target in air, Journal of Analytical Atomic Spectrometry, 23(11) (2008) 1518-1528.

[4] D.W. Hahn, N. Omenetto, Laser-induced breakdown spectroscopy (LIBS), part I: review of basic diagnostics and plasma-particle interactions: still-challenging issues within the analytical plasma community, Applied spectroscopy, 64(12) (2010) 335A-366A.

[5] I. Horn, M. Guillong, D. Günther, Wavelength dependant ablation rates for metals and silicate glasses using homogenized laser beam profiles - implications for LA-ICP-MS, Applied Surface Science, 182(1) (2001) 91-102.

[6] Q. Lu, S.S. Mao, X. Mao, R.E. Russo, Theory analysis of wavelength dependence of laserinduced phase explosion of silicon, Journal of Applied Physics, 104(8) (2008) 083301.

[7] C. Porneala, D.A. Willis, Effect of the dielectric transition on laser-induced phase explosion in metals, International journal of heat and mass transfer, 49(11) (2006) 1928-1936.

[8] Y. Wang, N. Shen, G.K. Befekadu, C.L. Pasiliao, Modeling pulsed laser ablation of aluminum with finite element analysis considering material moving front, International Journal of Heat and Mass Transfer, 113 (2017) 1246-1253.

[9] J.H. Yoo, S.H. Jeong, R. Greif, R.E. Russo, Explosive change in crater properties during high power nanosecond laser ablation of silicon, Journal of Applied physics, 88(3) (2000) 1638-1649.

[10] T. Butler, C. Dawson, T. Wildey, A posteriori error analysis of stochastic differential equations using polynomial chaos expansions, SIAM Journal on Scientific Computing, 33(3) (2011) 1267-1291.

[11] R. Ghanem, J. Red-Horse, Propagation of probabilistic uncertainty in complex physical systems using a stochastic finite element approach, Physica D: Nonlinear Phenomena, 133(1) (1999) 137-144.

[12] R.G. Ghanem, P.D. Spanos, Stochastic finite elements: a spectral approach, Courier Corporation, 2003.

[13] O.M. Knio, O.P. Le Maitre, Uncertainty propagation in CFD using polynomial chaos decomposition, Fluid Dynamics Research, 38(9) (2006) 616-640.

[14] H.N. Najm, Uncertainty quantification and polynomial chaos techniques in computational fluid dynamics, Annual review of fluid mechanics, 41 (2009) 35-52. 
[15] D. Xiu, G.E. Karniadakis, The Wiener--Askey polynomial chaos for stochastic differential equations, SIAM journal on scientific computing, 24(2) (2002) 619-644.

[16] H.G. Matthies, C.E. Brenner, C.G. Bucher, C.G. Soares, Uncertainties in probabilistic numerical analysis of structures and solids-stochastic finite elements, Structural safety, 19(3) (1997) 283-336.

[17] R. Ghanem, Probabilistic characterization of transport in heterogeneous media, Computer Methods in Applied Mechanics and Engineering, 158(3-4) (1998) 199-220.

[18] T.D. Hien, M. Kleiber, Stochastic finite element modelling in linear transient heat transfer, Computer Methods in Applied Mechanics and Engineering, 144(1-2) (1997) 111-124.

[19] O.P. Le Ma1tre, O.M. Knio, B.J. Debusschere, H.N. Najm, R.G. Ghanem, A multigrid solver for two-dimensional stochastic diffusion equations, Computer Methods in Applied Mechanics and Engineering, 192(41) (2003) 4723-4744.

[20] A. Gragossian, S.H. Tavassoli, B. Shokri, Laser ablation of aluminum from normal evaporation to phase explosion, Journal of Applied Physics, 105(10) (2009) 103304.

[21] B. Kim, R. Iida, D.H. Doan, K. Fushinobu, Nanosecond pulse laser scribing using Bessel beam for single shot removal of transparent conductive oxide thin film, International Journal of Heat and Mass Transfer, 107 (2017) 829-835.

[22] C. Porneala, D.A. Willis, Observation of nanosecond laser-induced phase explosion in aluminum, Applied physics letters, 89(21) (2006) 211121.

[23] G.M. Pound, Selected values of evaporation and condensation coefficients for simple substances, Journal of Physical and Chemical Reference Data, 1(1) (1972) 135-146.

[24] S. Tao, Y. Zhou, B. Wu, Y. Gao, Infrared long nanosecond laser pulse ablation of silicon: Integrated two-dimensional modeling and time-resolved experimental study, Applied Surface Science, 258(19) (2012) 7766-7773.

[25] P. Deierling, O.I. Zhupanska, C.L. Pasiliao, Micromechanical modeling of metal-ceramic composites for high temperature applications, in: 56th AIAA/ASCE/AHS/ASC Structures, Structural Dynamics, and Materials Conference, Kissimmee, Florida, 2015, pp. 0129.

[26] A.V. Gusarov, I. Smurov, Near-surface laser-vapour coupling in nanosecond pulsed laser ablation, Journal of Physics D: Applied Physics, 36(23) (2003) 2962.

[27] N. Shen, H. Ding, Q. Wang, H. Ding, Effect of confinement on surface modification for laser peen forming without protective coating, Surface and Coatings Technology, 289 (2016) 194-205.

[28] E. Assuncao, S. Williams, Effect of material properties on the laser welding mode limits, Journal of Laser Applications, 26(1) (2014) 012008.

[29] V. Morel, A. Bultel, B.G. Chéron, The critical temperature of aluminum, International Journal of Thermophysics, 30(6) (2009) 1853-1863. 
[30] B. Wu, Y.C. Shin, Absorption coefficient of aluminum near the critical point and the consequences on high-power nanosecond laser ablation, Applied physics letters, 89(11) (2006) 111902.

[31] ABAQUS User Subroutine Reference Manual, in: ABAQUS 6.14 Documentation.

[32] T. Homma, A. Saltelli, Importance measures in global sensitivity analysis of nonlinear models, Reliability Engineering \& System Safety, 52(1) (1996) 1-17. 